\title{
O enfermeiro na saúde indígena: uma revisão de literatura
}

\author{
The nurse in indigenous health: a literature review \\ La enfermera en salud indígena: revisión de la literatura
}

Recebido: 28/11/2021 | Revisado: 03/12/2021 | Aceito: 03/12/2021 | Publicado: 04/12/2021

\author{
Antonia Batista dos Santos \\ ORCID: https://orcid.org/0000-0002-4817-0805 \\ Centro Universitário da Amazônia, Brasil \\ E-mail: tanhabs200@gmail.com \\ Sâmia Lívia Miranda Cardoso \\ ORCID: https://orcid.org/0000-0002-8646-4062 \\ Centro Universitário da Amazônia, Brasil \\ E-mail: samiacardoso10@gmail.com \\ Maria da Conceição Caetano Siqueira \\ ORCID: https://orcid.org/0000-0003-1731-4076 \\ Centro Universitário da Amazônia, Brasil \\ E-mail: siqueira_44@hotmail.com
}

\begin{abstract}
Resumo
Entende-se que, assim como aqueles que não são indígenas, a população indígena também tem seus direitos à educação, a moradia, lazer, assim como à saúde, de acordo com a Constituição Federal de 1988. Objetiva -se sobre a atuação do enfermeiro na saúde indígena, dando destaque para a assistência de enfermagem nos serviços de saúde indígena. Estudo descritivo, revisão literária, utilizando artigos de periódicos científicos da área da saúde, teses e dissertações, disponíveis em: SCIELO, LILACS, MEDLINE; nos idiomas português, inglês e espanhol. Analisados pelo método de Bardin. A luta pela saúde indígena no Brasil, foi legitimada com a criação do Subsistema de Atenção à Saúde dos Povos Indígenas, através da Lei Arouca. Os profissionais envolvidos são definidos conforme a situação epidemiológica, necessidades de saúde, características geográficas, acesso e nível de organização dos serviços respeitando as especificidades étnicas de cada povo indígena, devendo atuar de forma articulada aos demais serviços do SUS. O perfil do profissional enfermeiro assim como a sua atuação, vai ganhando forma, de acordo com as referências que são encontradas dentro da área indígena que o mesmo atua, contribuindo para a consolidação de um modelo de atenção que vem a se afastar do que é proposto pelas diretrizes políticas existentes Os conhecimentos do enfermeiro a respeito de saúde indígena, são fundamentais para ofertar uma assistência de qualidade, no entanto, um fator que deve ser levado em consideração são as suas experiências a partir do momento que este começa a realizar seus trabalhos junto a essas populações.
\end{abstract}

Palavras-chave: Sistema de saúde indígena; Saúde de populações indígenas; Serviço de saúde indígena.

\begin{abstract}
It is understood that, as well as those who are not indigenous, the indigenous population also has their rights to education, housing, leisure, as well as health, in accordance with the Federal Constitution of 1988. in indigenous health, with emphasis on nursing care in indigenous health services. Descriptive study, literary review, using articles from scientific journals in the health field, theses and dissertations, available at: SCIELO, LILACS, MEDLINE; in Portuguese, English and Spanish. Analyzed by the method of Bardin. The struggle for indigenous health in Brazil was legitimized with the creation of the Health Care Subsystem for Indigenous Peoples, through the Arouca Law. The professionals involved are defined according to the epidemiological situation, health needs, geographic characteristics, access and level of organization of services, respecting the ethnic specificities of each indigenous people, and must act in conjunction with other SUS services. The profile of the professional nurse, as well as his performance, is taking shape, according to the references found within the indigenous area in which he works, contributing to the consolidation of a care model that moves away from what is proposed. according to existing policy guidelines Nurses' knowledge of indigenous health is essential to offer quality care, however, a factor that should be taken into account is their experiences from the moment they begin to carry out their work together. to these populations.
\end{abstract}

Keywords: Indigenous health system; Health of indigenous populations; Indigenous health service.

\section{Resumen}

Se entiende que, además de los que no son indígenas, la población indígena también tiene sus derechos a la educación, vivienda, esparcimiento, así como a la salud, de acuerdo con la Constitución Federal de 1988. en salud indígena, con énfasis en enfermería. atención en los servicios de salud indígenas. Estudio descriptivo, revisión literaria, utilizando artículos de revistas científicas del campo de la salud, tesis y disertaciones, disponible en: SCIELO, LILACS, MEDLINE; en portugués, inglés y español. Analizado por el método de Bardin. La lucha por la salud indígena en Brasil se legitimó con la creación del Subsistema de Atención a la Salud de los Pueblos Indígenas, a través de la Ley 
Arouca. Los profesionales involucrados se definen de acuerdo a la situación epidemiológica, necesidades de salud, características geográficas, acceso y nivel de organización de los servicios, respetando las especificidades étnicas de cada pueblo indígena, debiendo actuar en conjunto con los demás servicios del SUS. El perfil del enfermero profesional, así como su desempeño, va tomando forma, de acuerdo con los referentes encontrados dentro del área indígena en la que trabaja, contribuyendo a la consolidación de un modelo de atención que se aleja de lo propuesto. lineamientos de política El conocimiento de las enfermeras sobre la salud indígena es fundamental para ofrecer una atención de calidad, sin embargo, un factor que se debe tomar en cuenta son sus vivencias desde el momento en que comienzan a realizar su trabajo en conjunto a estas poblaciones.

Palabras clave: Sistema de salud indígena; Salud de las poblaciones indígenas; Servicio de salud indígena.

\section{Introdução}

O Brasil é reconhecido por sua diversidade, não apenas por sua fauna, flora e iguarias culinárias, mas também por sua diversidade cultural, racial e religiosa. Neste cenário não se pode deixar de destacar os tradicionais, dentre eles os povos indígenas, de acordo cuja o censo populacional em 2010 mostrava um total de 810 mil habitantes indígenas distribuídos em zonas rurais e zonas urbanas. (Ibge, 2021).

Entende -se que, assim como aqueles que não são indígenas, a população indígena também tem seus direitos à educação, a moradia, lazer, assim como à saúde, de acordo com a Constituição Federal de 1988:

Os indígenas são cidadãos, possuem todos os direitos do cidadão comum, além daqueles específicos garantidos pela Constituição. São garantidos o respeito à sua organização social, costumes, crenças e tradições, e os direitos originários sobre as terras que tradicionalmente ocupam, competindo à União demarcá-las, protegê-las e fazer respeitar todos os seus bens. Essa mesma Constituição define a saúde como direito de todos e dever do Estado, consolidando os princípios para a criação do Sistema Único de Saúde/SUS. (BRASIL, 1988).

Sendo a população indígena reconhecida através de seus direitos, como cidadãos brasileiros, essa mesma constituição lhes dá direito à saúde, e acesso igualitário aos serviços de saúde disponibilizados aos demais cidadãos, de acordo com os princípios do SUS, a integralidade, universalidade e a equidade; confirmando o que rege a constituição federal no que diz respeito a saúde (Brasil, 2017):

Art. 196. A saúde é um direito de todos (ou seja, é um direito universal, faz alusão ao princípio do SUS) e dever do Estado, garantido mediante políticas sociais e econômicas que visemà redução de risco de doença e de outros agravos. E ao acesso universal e igualitário às ações e serviços para sua promoção, proteção e recuperação (Brasil, 2017).

Para que sejam respeitados esses direitos, a se fez necessária a criação de um subsistema de atenção aos povos indígenas, afim de atender as suas essas populações em suas necessidades, bem como nas suas peculiaridades, uma vez que tais povos costumam estar em lugares afastados, ter seus costumes, modos de vida e linguagem (Funai, 2021).

Diante do exposto esta obra tem por objetivo discorrer sobre a atuação do enfermeiro na saúde indígena, dando destaque para a assistência de enfermagem nos serviços de saúde indígena, à luz das literaturas.

\section{Metodologia}

Trata se de um estudo descritivo, do tipo revisão de literatura, cuja construção utilizou - se de artigos de periódicos científicos da área da saúde, e protocolos de instituições de saúde disponíveis à comunidade, assim como teses e dissertações, disponíveis nas seguintes bases de dados científicos: SCIELO (Scientific Eletronic Library Online), LILACS (Literatura Latino - Americana e do Caribe em Ciências da Saúde), MEDLINE (Medical Literature Analysis and Retrieval System Online); nos idiomas português, inglês e espanhol; usando como descritores: sistema de saúde indígena, saúde de populações indígenas, serviço de saúde indígena.

Quanto ao aspecto descritivo desta obra, Gerhardt e Silveira (2009), corroboram que este tipo de estudo se constitui uma das classificações das pesquisas científicas cujo o objetivo é descrever as características, sejam elas de uma população, 
um fenômeno, aspectos documentais ou experiência para um estudo mais completo.

Para os critérios de inclusão, foi considerado: ser escrito nos idiomas anteriormente citados, ter relação com o tema, ter sido publicados entre os anos de 2001 a 2021; quanto critérios de exclusão: textos incompletos, versão apenas em resumos, textos sem referência à autoria e não atender aos critérios de inclusão.

Para a análise de dados obtidos, levou -se em consideração a obra de Laurence Bardin, literatura de referência atual para análise de conteúdo. De acordo com Bardin (2012), a análise de conteúdo trata -se de um conjunto de técnicas de análise de comunicações, que se utiliza de procedimentos sistemáticos e objetivos de descrição do conteúdo das mensagens. Azevedo et al. (2021), sintetiza as fases de análise de dados (Figura 1).

Figura 1. Síntese do método de análise de conteúdo de Bardin.

\begin{tabular}{c|l}
\hline \multicolumn{2}{c}{ MÉTODO DE ANÁLISE DE CONTEÚDO } \\
\hline $\begin{array}{c}\text { FASE I } \\
\text { Pré - análise }\end{array}$ & $\begin{array}{l}\text { Seleção dos materiais que tem relação com o objeto de estudo, embasamento argumentativos } \\
\text { para os resultados obtidos. Nesta fase são aplicados os critérios de inclusão e exclusão, para } \\
\text { facilitar a obtenção de dados que serão realmente aproveitados. }\end{array}$ \\
\hline $\begin{array}{c}\text { FASE II } \\
\text { Exploração do } \\
\text { material }\end{array}$ & $\begin{array}{l}\text { Estudo e leitura minuciosa dos materiais obtidos. Nesta fase são avaliação os escritos e } \\
\text { selecionados os que serão necessários para sustentar do tema de estudo, assim como os que } \\
\text { serão utilizados para fazer correlação com a proposta do tema em questão. }\end{array}$ \\
\hline $\begin{array}{c}\text { FASE III } \\
\text { Tratamento dos } \\
\text { resultados }\end{array}$ & $\begin{array}{l}\text { Validação dos dados analisados. Relação dos objetivos com os resultados, e possível } \\
\text { surgimento de novas hipóteses. }\end{array}$ \\
\hline
\end{tabular}

Fonte: Azevedo et al. (2021).

As fases de análises propostas pela autora e utilizadas neste estudo foram respectivamente: pré - análise, exploração sistemática dos documentos e tratamento dos resultados. Os passos estão descritos a seguir:

I - Pré - análise: Fase onde foram coletados os artigos, ou seja, a escolha dos materiais que estão interligados ao objeto da pesquisa, bem como do seu referencial, que dar argumentos aos resultados encontrados.

II - Exploração do material: fase de seleção, correspondente à análise propriamente dita do conteúdo dos artigos, ou seja, estudo e leitura dos dados coletados. Após foi realizada a codificação do material com o objetivo. Também foi nessa fase que se verificou toda a documentação necessária que dá sustentação ao problema a ser estudado.

III - Tratamento dos resultados: foi a fase da validação dos resultados analisados. Para a validação, foi imprescindível analisar a fidedignidade destes artigos, buscando as fontes, autoria e ano de publicação. Após relacionados os objetos com os resultados encontrados, foi possível partir para novas hipóteses, dando origem novas propostas de pesquisa, através das conclusões obtidas.

\section{Resultados e Discussão}

Inicialmente foram selecionados 25 artigos usando os seguintes descritores acima mencionados, dos quais apenas 8 estavam de acordo com os critérios de inclusão. Estes estão dispostos na Tabela 1. 
Tabela 1. Artigos selecionados para discussão.

\begin{tabular}{|c|c|c|}
\hline AUTOR/DATA & \begin{tabular}{|c|} 
TÍTULO DO ARTIGO \\
\end{tabular} & $\begin{array}{r}\text { OBJETIVO } \\
\end{array}$ \\
\hline Martins (2017) & $\begin{array}{l}\text { O trabalho do enfermeiro na saúde indígena: } \\
\text { desenvolvendo competênciaspara a atuação no } \\
\text { contexto intercultural }\end{array}$ & $\begin{array}{l}\text { Analisar a vivência do trabalho de saúde dentro do territórioindígena } \\
\text { como um espaço potencial de aprendizagem para que o enfermeiro } \\
\text { qualifique sua prática profissional voltada para a atuação neste } \\
\text { contexto intercultural. }\end{array}$ \\
\hline Ribeiro et al.(2015). & $\begin{array}{l}\text { Trabalho de Enfermagem em uma Instituição } \\
\text { de Apoio ao Indígena }\end{array}$ & $\begin{array}{l}\text { Analisar o processo de trabalho da enfermagem em uma instituição } \\
\text { de saúde indígena. Para tal, pesquisou-se uma instituição do estado } \\
\text { de Mato Grosso do Sul e focou-se } \\
\text { especialmente nos instrumentos do processo de trabalho. }\end{array}$ \\
\hline Rocha at al. (2019). & $\begin{array}{l}\text { A luta dos povos indígenas por saúde em } \\
\text { contextos de conflitos ambientais noBrasil }\end{array}$ & $\begin{array}{l}\text { Apresentar um panorama dos conflitos socioambientais envolvendo } \\
\text { os povos indígenas brasileiros, suas estratégias para garantir o } \\
\text { acesso e a qualidade do Subsistema de Atenção à Saúde Indígena } \\
\text { (SASI), e alternativas que eles } \\
\text { têm proposto para o enfrentamento dos problemas gerados }\end{array}$ \\
\hline Rissardo et al.(2014). & $\begin{array}{l}\text { Práticas de cuidado ao idoso indígena - atuação } \\
\text { dos profissionais de saúde }\end{array}$ & $\begin{array}{l}\text { Este estudo objetivou compreender as práticas de cuidado dos } \\
\text { profissionais de saúde que assistem os idosos Kaingang }\end{array}$ \\
\hline Santos et al. (2011). & $\begin{array}{l}\text { Assistência prestada pelo Sistema Único de } \\
\text { Saúde de Teresina à população indígena do } \\
\text { Maranhão }\end{array}$ & $\begin{array}{l}\text { Descrever o acesso aos serviços de saúde pelos índios das etnias } \\
\text { Kanela e Guajajara e sua satisfação com a assistênciaprestada pelo } \\
\text { Sistema Único de Saúde (SUS). }\end{array}$ \\
\hline Silva et al. (2021). & $\begin{array}{l}\text { Dificuldades vivenciadas pelos profissionais } \\
\text { de saúde no atendimento à população indígena }\end{array}$ & $\begin{array}{l}\text { Descrever sobre as dificuldades vivenciadas no atendimentopelos } \\
\text { profissionais de saúde no atendimento à população } \\
\text { indígena }\end{array}$ \\
\hline Viana et al. (2020). & $\begin{array}{l}\text { A atuação do enfermeiro na saúde indigena: } \\
\text { uma análise integrativa da literatura }\end{array}$ & $\begin{array}{l}\text { Investigar a atuação do enfermeiro na saúde indígena,segundo a } \\
\text { percepção da literatura. }\end{array}$ \\
\hline Iczenovicz(2018). & $\begin{array}{l}\text { Saúde Indígena: Reflexões Contemporâneas. } \\
\text { Caderno Ibero-Americano }\end{array}$ & $\begin{array}{l}\text { O estudo analisar alguns elementos da trajetória sócio- histórica das } \\
\text { políticas públicas em saúde no processo de efetivação e } \\
\text { disponibilização deste direito fundamental } \\
\text { social junto as Comunidades Indígenas do Brasil. }\end{array}$ \\
\hline
\end{tabular}

Fonte: Santos, Cardoso e Siqueira (2021).

Após a leitura dos artigos selecionados surgiram as vertentes que serão discutidas a seguir:

\subsection{Saúde indígena no Brasil}

Em suas reflexões sobre a saúde indígena no Brasil, Wenczenovicz (2018) afirma que:

Em se tratando de comunidades vulneráveis no Brasil é possível apontar que essas vivenciam situações de exclusão, invisibilidade e discriminação que, em última instância, as colocam em posição de maior vulnerabilidade frente a uma série de agravos. Corroboram com essa afirmação os coeficientes de morbi-mortalidade mais altos do que os registrados em nível nacional; fome e desnutrição, riscos ocupacionais e violência física e simbólica que são apenas alguns dos múltiplosreflexos sobre a saúde decorrentes da minimização social (Wenczenovicz, 2018).

A luta pela saúde indígena no Brasil, foi mais legitimada com a criação do Subsistema de Atenção à Saúde dos Povos Indígenas, que foi criado em 1999, através da Lei $n^{\circ}$ 9.836/99, mais conhecida como Lei Arouca. Este subsistema é composto pelos Distritos Sanitários Especiais Indígenas- DSEIS, que se constituem em uma rede de serviços implantada nos territórios 
indígenas com o objetivo de atender essa população, levando em consideração os critérios geográficos, demográficos e culturais.

Á Luz dos princípios do SUS, o Subsistema de Atenção à Saúde dos Povos Indígenas considera que a participação indígena é uma premissa fundamental para o melhor controle e planejamento dos serviços, bem como uma forma de reforçar a autodeterminação desses povos.

De acordo com a Fundação Nacional do Índio - FUNAI (2021), para garantir aos povos indígenas o acesso igualitário e atenção integral aos povos indígenas de acordo com os princípios do SUS, se fez necessário o estabelecimento de algumas diretrizes que orientam a definição de instrumentos de planejamento, implementação, avaliação e controle das ações de atenção à saúde dos povos indígenas, estas estão dispostas no (Quadro 1).

Quadro 1. Compilado de orientações para atenção á saúde indígena.

\begin{tabular}{|l|}
\hline \multicolumn{1}{|c|}{ DIRETRIZES PARA AÇÕES DE ATENÇão AOS POVOS INDÍGENAS } \\
\hline 1- Organização dos serviços de atenção à saúde dos povos indígenas na forma de Distritos Sanitários Especiais e Pólos-Base,no \\
nível local, onde a atenção primária e os serviços de referência se situam; \\
2 - Preparação de recursos humanos para atuação em contexto intercultural; \\
3 - Monitoramento das ações de saúde dirigidas aos povos indígenas; \\
4 - Articulação dos sistemas tradicionais indígenas de saúde; \\
5 - Promoção do uso adequado e racional de medicamentos; \\
6 - Promoção de ações específicas em situações especiais; \\
7 - Promoção da ética na pesquisa e nas ações de atenção à saúde envolvendo comunidades indígenas; \\
$\mathbf{8}$ - Promoção de ambientes saudáveis e proteção da saúde indígena; \\
$\mathbf{9}$ - Controle social.
\end{tabular}

Fonte: Santos, Cardoso e Siqueira (2021).

Além dos aspectos organizacionais do serviço de saúde indígena, também devem ser levados em consideração alguns fatores peculiares a essa população como, a sua situação de transculturação, a localização geográfica dos índios, com ênfase nas dificuldades de acesso a essas aldeias; tais fatores acabam por se soma a falta de infraestrutura e recursos (Santos et al., 2016).

No entanto, mesmo com as tentativas de inclusão e assistência melhorada a esses povos, ainda é possível observar com frequência a desigualdade no suporte à rede de saúde indígena. Rocha et al. (2019), em sua obra sobre a luta dos povos indígenas por saúde, observaram que, além dos pontos destacados anteriormente é a condição desfavorável na manutenção do processo saúde-doença dos povos indígenas; explica que a vulnerabilidade e desigualdade em relação a assistência a que é oferecida a população nacional, ainda que sejam comparados com a parcela mais pobre da sociedade brasileira.

Os mesmos autores anteriormente citados, também corroboram que, os polos-bases nas aldeias são estruturados apenas para atender a atenção primária, dessa forma os casos de média e alta complexidade acabam sendo referenciados para as unidades do Sistema Único de Saúde - SUS, tais serviços são localizados em centros urbanos próximos ou em outros municípios, onde os povos indígenas sofrem com diferenças organizacionais, culturais, linguística, refletindo negativamente na possibilidade do acesso e na qualidade do cuidado ofertado, piorando o processo saúde - doença que se encontra (Rocha et al., 2019).

\subsection{A equipe de saúde indígena}

A composição da Equipe Multiprofissional de Saúde Indígena - EMSI (Quadro 2), é regulamentada pela portaria no 1. 317, de 3 de agosto de 2017. Nela consta que, a EMSI corresponde a um conjunto de profissionais responsáveis pela atenção 
básica à saúde indígena em uma área, sob gestão do Subsistema de Atenção à Saúde Indígena (SASI-SUS) (Brasil, 2017).

No que diz respeito aos tipos de estabelecimentos nos quais as EMSI poderão ser cadastradas, a portaria estabelece o seguinte: Posto de Saúde, Centro de Saúde/Unidade Básica ou Unidade de Atenção à Saúde Indígena, em qualquer de seus subtipos, exceto Casa à Saúde Indígena - CASAI.

Em relação ao quantitativo e categorias dos profissionais fazem parte das EMSI, estes são definidos conforme a situação epidemiológica, a necessidades de saúde, as características geográficas, o acesso e nível de organização dos serviços respeitando as especificidades étnicas e culturais de cada povo indígena, devendo atuar de forma articulada e integrada aos demais serviços do SUS.

Quadro 2. Composição da Equipe Multiprofissional de Saúde Indígena - EMSI.

\begin{tabular}{|c|c|}
\hline DESCRIÇÃO DA OCUPAÇÃO & QUANTIDADE EQUIPE MÍNIMA \\
\hline Médico clínico ou enfermeiro (NS) & 01 \\
\hline Técnico de enfermagem ou auxiliar deenfermagem (NM) & 01 \\
\hline $\begin{array}{c}\text { Agente indígena de saúde ou agente indígena desaneamento } \\
\text { (NM) }\end{array}$ & 01 \\
\hline
\end{tabular}

Legendas: NS (Nível superior), NM (Nível médio). Fonte: Santos, Cardoso e Siqueira (2021).

O gestor/coordenador local pode, opcionalmente, além da equipe mínima, incluir os seguintes profissionais as equipes que prestam atenção à saúde indígena como: médicos clínicos (família), cirurgião dentista (família), enfermeiros (família), técnicos e auxiliares de enfermagem (família), técnico em saúde bucal, auxiliar em saúde bucal, trabalhadores em serviços de promoção e apoio à saúde (família), agente de saúde pública, microscopista, entre outros. Estes não deverão ser marcados como equipe mínima (Brasil, 2017).

\subsection{A importância do papel do enfermeiro na saúde indígena}

Para Fernandes e Simpson (2016), o enfermeiro que se compromete com a assistência na saúde indígena deve ser capaz de se familiarizar com as peculiaridades do subsistema de saúde, assim como, buscar entender de modo holístico como a comunidade que ele está inserido, responde as situações de saúde e doença.

De acordo com o estudo proposto por Martins (2017), o perfil do profissional enfermeiro assim como a sua atuação, vai ganhando forma, de acordo com as referências que são encontradas dentro da área indígena que o mesmo atua, contribuindo para a consolidação de um modelo de atenção que vem a se afastar do que é proposto pelas diretrizes políticas existentes. Dessa forma, se faz necessário que o enfermeiro mantenha - se atualizado constantemente, através da educação permanente (Oliveira \& Ravelli, 2020).

Em um estudo conduzido por Rissardo et al. (2014) a respeito da atuação da equipe multiprofissional em saúde indígena, realizado com funcionários da EMSI (Equipe Multidisciplinar de Saúde Indígena) de Angra dos Reis, observaram que apesar da obrigatoriedade de atualização, capacitação e aperfeiçoamento dos cuidados assistências de saúde, por meio da Política Nacional da População Indígena, além de ser dever conforme o próprio código de ética do profissional; dos 10 componentes da equipe em pesquisa, somente dois profissionais relataram algum tipo de preparo para introdução ao trabalho assistencial ao indígena os demais, mencionaram a falta de educação permanente anual por parte dos gestores.

O fato acima citado, também se confirma na obra de Ribeiro et al. (2015), destacaram a falta de capacitação ao ingressarem no posto de trabalho na Casai (Casa de Apoio ao Índio). O mesmo autor, ainda ressalta que esse percalço se repete em diferentes localidades de atenção à saúde indígena.

Vale ressaltar que a pluralidade cultural é um fator que deve ser levado em consideração, devendo o profissional de 
enfermeiro se moldar de acordo com a comunidade que está inserido, afim de ganhar a confiança de seus pacientes, para das prosseguimento a suas atribuições. As palavras de Mendonça (2010), nos traz uma breve reflexão a respeito da prática

Conhecer o diferente passa a ser uma reflexão sobre a nossa própria prática, confirma a necessidade de se dispor a ouvir, se dispor ao diálogo. Essa é uma das habilidades mais importantes que os profissionais de saúde que atuam em saúde indígena devem exercitar e construir. Quando nos dispomos a ouvir, não só a ouvir, mas escutar, e exercer nosso papel de interlocutores, estamos trabalhando com a perspectiva de repensar nossa própria cultura, relativizar nossos próprios paradigmas (Mendonça, 2010).

Dentre os fatores que limitam a atuação do enfermeiro nas áreas indígenas são o espaço físico para a realização de procedimentos, situações de conflito ocorridas em ambiente de trabalho devido ao diálogo e à necessidade de negociação com os especialistas tradicionais (parteiras, xamãs, pajés e outros), assim como a negociação com as famílias na tomada de decisões em situações de urgência e emergência (Silva et al. 2021).

Em sua obra sobre as competências a serem desenvolvidas pelo enfermeiro para a atuação em localidades indígenas, Martins (2017), corrobora que outro fator a ser considerado é a fragilidade dos limites legais de atuação profissional, para o trabalho dentro de áreas indígenas. Tal fragilidade também foi apontada na III Conferência Nacional de Saúde Indígena (Brasil, 2001).

De acordo com (Viana et al. 2020), outro fato observado é o atendimento periódico e descontínuo, pois por muitas vezes o enfermeiro não reside na aldeia, tendo seu trabalho feito em regime de escala, uma determinada quantidade de dias na aldeia e aproximadamente 10 dias de folga. Esta rotatividade, não somente por parte do enfermeiro, acaba por levar os indígenas a buscarem assistência fora das aldeias, ou seja, nos centros de saúde da rede municipal. Havendo articulação entre a equipe de saúde indígena e os demais serviços da rede de saúde do Sistema Único de Saúde.

No que se refere aos registros de enfermagem, Ribeiro et al. (2015) afirmam que que o registro de enfermagem desempenha papel essencial no trabalho da enfermagem. No entanto, Viana et al. (2020), observaram que os profissionais de enfermagem têm visto os registros como um processo mecânico e maçante buscando apenas cumprir as regras, objetivos e finalidade, não como um Instrumento que possibilita a continuidade do cuidado integral ao indígena. Os mesmos autores ainda salientam que até as ações de saúde tem sido percebidas somente como normas a serem cumpridas na percepção do enfermeiro.

\section{Considerações Finais}

Nos períodos anteriores à 2017 não foram encontradas muitas literaturas que tratassem acerca da experiência da enfermagem nos serviços de saúde indígena; porém, nos anos seguintes foi observado que ocorreram mais publicações a respeito.

A partir dos anos de 2017 e atualmente, surgiram mais experiências, descrevendo a realidade e os desafios para o trabalho da enfermagem na saúde indígena; este fato reforça a maior sensibilização por parte dos profissionais de saúde, comunidade científica e gestores, afim de preencher a lacuna no conhecimento desse assunto tão necessário e atual.

Os conhecimentos do enfermeiro a respeito de saúde indígena, são fundamentais para ofertar uma assistência de qualidade, no entanto, um fator que deve ser levado em consideração são as suas experiências a partir do momento que este começa a realizar seus trabalhos junto a essas populações. O conhecimento científico, aliado à experiência humana com a cultura peculiar desses povos, é que faz o verdadeiro profissional.

Faz-se necessário criar estratégias de ações de saúde que levem em consideração a realidade dos povos indígenas, principalmente aqueles que vivem em regiões mais remotas, que por vezes são afastadas dos grandes centros urbanos, e consequentemente, onde a saúde básica acaba por ser negligenciada, isto tanto por falta de recursos estruturais e materiais, 
como quanto a recursos humanos capacitados.

Observou se também a escassez de estudos recentes voltados para o tema desta obra, desse modo, sugere - se um olhar mais atento a respeito das experiências da enfermagem na saúde indígena no Brasil.

\section{Referências}

Azevedo, D. K. L., Silva, C. M. P., \& Maia, A. L. (2021). O papel da gestão de enfermagem na implementação da meta de cirurgia segura: uma revisão de literatura. Research, Society and Development, 10(14), e584101422711, http://dx.doi.org/10.33448/rsd- v10i14.22711.

Bardin, L. Análise de conteúdo: a revisão de Laurence Bardin. (2012). Revista Eletrônica de Educação. Programa de Pós-graduação em Educação. 6(1). http://dx.doi.org/10.14244/\%2519827199291.

Brasil. (1988). Constituição (1988). Constituição da República Federativa do Brasil. Presidência da República, Casa Civíl. http://www.planalto.gov.br/ccivil _03/constituicao/constituicao.htm

Brasil. (2001). Ministério da Saúde. III CONFERÊNCIA NACIONAL DE SAÚDE INDÍGENA - Relatório Final. Luziânia, GO, 14 a 18/06/2001. Conselho Nacional de Saúde Indígena. https://bvsms.saude.gov.br/bvs/publicacoes/3_conferencia_nacional_saude_indigena_relatorio_final.pdf.

Brasil. (2017). Artigo 196. Constituição Federal (Texto compilado até a Emenda Constitucional no 96 de 06/06/2017). Conselho Nacional de Saúde. 2017. https://www.senado.leg.b r/atividade/const/con1988/con1988_06.06.2017/art_196_.asp.

Brasil. (2017). Ministério da Saúde. Portaria no 1. 317, de 3 de agosto de 2017. Adequa o registro das informações relativas a estabelecimentos que realizam ações de Atenção à Saúde para populações Indígenas no CNES. Secretaria de atenção à saúde. https://bvsms.saude.gov.br/bvs/saudelegis/sas/2017/prt1317_08_08_2017.html.

Fernandes, M. N. F., \& Simpson, C. A. (2016). Saúde indígena: experiência de enfermagem com a etnia Munduruku. Biblioteca Lascasas. 12(2). http://www.index-f.com/lascasas/documentos/lc0900.

Funai. (2021). Fundação Nacional do Índio. Saúde: Subsistema de Atenção à Saúde dos Povos. Ministério da Justiça e Segurança Pública. http://www.funai.gov.br/index.php/saude.

Gerhardt, T. E., \& Silveira, D. T. (2009). Métodos de pesquisa. Planejamento e gestão para o desenvolvimento rural da SEAD/UFRGS. Universidade Federal do Rio Grande do Sul. https://lume.ufrgs.br/bitstream/handle/10183/52806/000728684.pdf?sequence=1\&isAllowed=y.

Ibge. (2010). Populações indígenas. Instituto Brasileiro de Geografia e Estatística. Ministério da Saúde. http://indigenas.ibge.gov.br/graficos-e-tabelas- 2.html

Martins, J. C. L. (2017). O trabalho do enfermeiro na saúde indígena: desenvolvendo competências para a atuação no contexto intercultural. Dissertação. Faculdade de saúde pública da Universidade de São Paulo -USP. https://www.teses.usp.br/teses/disponiveis/6/6135/tde-29082017- 152141/publico/Juli anaClaudiaLealMartinsORIGINAL.pdf.

Mendonça, S. B. M. (2010). Saúde indígena: distâncias que aproximam. In: Brasil. Ministério da Saúde. Secretaria de Atenção à Saúde. Política Nacional de Humanização. Cadernos Humaniza SUS, v.2, Brasília, DF, 2010, 179-194. Série B. Textos básicos de saúde.

Oliveira, A. G., \& Ravelli, R. C. R. (2020). Papel Do Enfermeiro Da Atenção Básica Na Assistência Na Saúde Indígena. Faculdade do Baixo Paraíba - FAP http://www.fap.com.br/anais/congresso-multidisciplinar-2020/comunicacao-oral/061.pdf

Ribeiro, A. A., Fortuna, C. M., \& Arantes, C. I. S. (2015). O Trabalho de Enfermagem em uma Instituição de Apoio ao Indígena. Texto Contexto Enfermagem, Florianópolis, 24(1), 138-145.

Rissardo, L. K., Alvim, N. A. T., Marcon, S. S., \& Carreira, L. (2014). Práticas de cuidado ao idoso indígena - atuação dos profissionais de saúde. Revista Brasileira de Enfermagem - REBE. 67(6), 919-927. https://doi.org/10.1590/0034-7167.2014670609

Rocha, D. F., Porto, M. F. S., \& Pacheco, T. (2019). A luta dos povos indígenas por saúde em contextos de conflitos ambientais no Brasil (1999-2014). Revista Ciência \& Saúde Coletiva. 2019; 24 (2):383-392. https://doi.org/10.1590/1413-81232018242.27972016

Santos, M. M., Cruz, K. J. C., Sá, L. C. R., Batista, C. C., Aguiar, E. M. G., \& Nogueira, A. M. T. (2011). Assistência prestada pelo Sistema Único de Saúde de Teresina à população indígena do Maranhão, 2011: um estudo descritivo. Revista Epidemiolgia Serviço de Saúde, 25(1). https://doi.org/10.5123/S167949742016000100013

Silva, E. C., Silva, Niedja C. D. L., Café, L. A., Almeida, P. M. O., Souza, L. N., \&Amanda, D. da S. (2021). Dificuldades vivenciadas pelosprofissionais de saúde no atendimento à população indígena. Revista Eletrônica Acervo Saúde / Electronic Journal Collection Health..13(1) e 54132021. https://doi.org/10.2524 8/reas.e5 413.2021.

Viana, J. A., Cipriano, D. M., Oliveira, M. C., Carneiro, A. M. C. T., Ribeiro, R. S., Feitosa, M. O., Cavalcante, M. D. S., Belfort, M. G. S., \& Santos, F. D. R. P. (2020). A atuação do enfermeiro na saúde indigena: uma análise integrativa da literatura. Brazilian Journal of health Review., 3(2), 2113-2127 $10.34119 /$ bjhrv3n2-065

Wenczenovicz, T. J. (2018). Saúde Indígena: Reflexões Contemporâneas. Caderno Ibero-Americano. Diretoria Sanitária, 7(1):63-82, http://dx.doi.org/10.1 7566/ciad s.v7i1.428. 